\title{
EFEITO FISIOLÓGICO DE INSETICIDAS E FUNGICIDA SOBRE A GERMINAÇÃO E VIGOR DE SEMENTES DE SOJA (Glycine max L.)
}

\section{PHYSIOLOGICAL EFFECT OF INSECTICIDES AND FUNGICIDES ON THE GERMINATION AND EFFECT OF SOYBEAN SEEDS (Glycine max L.)}

\author{
Estevam Matheus Costa ${ }^{1}$, Bruno de Moraes Nunes ${ }^{2}$, Matheus Vinicius Abadia Ventura ${ }^{3}$, Bruno \\ Henrique Tondato Arantes ${ }^{4}$, Gabriel Ribeiro Mendes ${ }^{5}$ \\ ${ }^{1}$ Mestrando em Ciências Agrárias, Instituto Federal Goiano - estevammcosta@yahoo.com.br \\ 2 Doutorando em Agronomia na Universidade Federal de Uberlândia - brunodemoraesnunes@gmail.com \\ ${ }^{3}$ Mestrando em Ciências Agrárias, Instituto Federal Goiano - matheusvinicius10@hotmail.com \\ ${ }^{4}$ Mestrando em Ciências Agrárias, Instituto Federal Goiano - bhtondato@gmail.com \\ ${ }^{5}$ Graduação em Agronomia pela Universidade do Estado de Minas Gerais -gmendes856@gmail.com
}

\begin{abstract}
Resumo: A proteção inicial das plantas por meio da utilização de inseticidas e fungicidas tem se mostrado eficiente para um bom manejo de pragas e doenças, entretanto algumas moléculas utilizadas no tratamento de sementes vêm demonstrando um efeito fisiológico sobre a germinação e o desenvolvimento vegetativo inicial. O presente trabalho teve como objetivo a avaliação do efeito fisiológico provocado por inseticidas contendo os princípios ativos Imidacloprido, Tiametoxam, e um fungicida com os princípios ativos Metalaxyl-M + Fludioxonil sobre a germinação e sobre o vigor das sementes. O experimento foi conduzido no Laboratório de Análise de Sementes da Universidade do Estado de Minas Gerais - Unidade Ituiutaba em Delineamento em Blocos Casualisados com seis tratamentos e quatro repetições para cada tratamento. Os tratamentos foram os seguintes: T1 - água destilada; T2 - inseticida Rocks ${ }^{\circledR}$ (Bifentrina + Imidacloprido); T3 - inseticida Cruiser ${ }^{\circledR}$

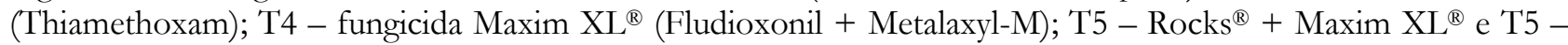
Cruiser $^{\circledR}+$ Maxim XL ${ }^{\circledR}$. A aplicação dos tratamentos foi realizada conforme recomendação dos fabricantes e foram avaliados o porcentual de germinação das sementes, o índice de velocidade de emergência, a condutividade elétrica da solução de embebição e o comprimento total de plântulas. Os dados foram submetidos à Análise de Variância $(0,05)$ e as médias foram comparadas pelo teste de Tukey $(0,05)$. Os resultados indicaram que não houve diferença estatisticamente significativa para as variáveis germinação (\%), índice de velocidade de emergência, condutividade elétrica da solução de embebição. Para a variável comprimento de plântulas constatou-se que houve diferença estatisticamente significativa ao nível de 5\%, sendo assim, os tratamentos 3,4 , 5 e 6 foram estatisticamente superiores aos tratamentos 1 e 2 .
\end{abstract}

Palavras chave: Tratamento de sementes; Thiamethoxam; Imidacloprido; Glycine max L.

\begin{abstract}
The initial protection of the plants through the use of insecticides and fungicides has been shown to be efficient for a good management of pests and diseases, however some molecules used in the treatment of seeds have demonstrated a physiological effect on germination and initial vegetative development. The objective of this work was to evaluate the physiological effect of insecticides containing the active ingredients Imidacloprid, Tiametoxam and a fungicide with the active principles Metalaxyl-M + Fludioxonil on germination and seed vigor. The experiment was conducted at the Laboratory of Seed Analysis of the University of the State of Minas Gerais - Ituiutaba Unit in Outline Design with 6 treatments and 4 replicates for each treatment. The treatments were as follows: T1 - distilled water; T2 - Rocks ${ }^{\circledR}$ insecticide (Bifenthrin + Imidacloprid); T3 Cruiser ${ }^{\circledR}$ insecticide (Thiamethoxam); T4 - Maxim XL ${ }^{\circledR}$ fungicide (Fludioxonil + Metalaxyl-M); T5 - Rocks ${ }^{\circledR}+$ Maxim XL ${ }^{\circledR}$ and $T 5$ - Cruiser ${ }^{\circledR}+$ Maxim XL ${ }^{\circledR}$. The application of the treatments was performed according to the manufacturers' recommendations and the percentage of seed germination, the rate of emergence, the electric conductivity of the soaking solution and the total length of seedlings were evaluated. The data were submitted to Analysis of Variance (0.05) and the means were compared by the Tukey test (0.05). The results indicated that there was no statistically significant difference for the variables germination (\%), emergency speed index, electrical conductivity of the imbibition solution. For the variable length of seedlings, it was found that there was a statistically significant difference at the $5 \%$ level, thus, treatments 3, 4, 5 and 6 were statistically superior to treatments 1 and 2 .
\end{abstract}

Keywords: Seed treatment; Thiamethoxam; Imidacloprid; Glycine max L. 


\section{INTRODUÇÃO}

O aumento da produção de soja e sua consequente expansão nos últimos anos, associada ao mono cultivo, favoreceu em grande escala o aumento da incidência de pragas $\mathrm{e}$ doenças, que podem atacar durante todas as fases da cultura, podendo assim reduzir significativamente a produtividade dessa oleaginosa, limitando a lucratividade e o êxito da produção.

A semente não pode ser considerada como um grão que germina. Ela possui atributos de qualidade genética, física, fisiológica e sanitária que um grão não tem e que lhe confere a garantia de um desempenho agronômico, que é a base fundamental para o sucesso de uma lavoura tecnicamente bem instalada.

Para evitar possíveis perdas decorrentes das ações de pragas e doenças do solo e da parte aérea, que causam danos as sementes e as plantas jovens, tem-se como alternativa, a utilização de inseticidas e fungicidas, com ação sistêmica, no tratamento de sementes. Esse tipo de tratamento apresenta custos mais baixos, impactos ambientais reduzidos, menor risco de intoxicação para o aplicador de produtos, maior rapidez e eficiência de aplicação, além de maior economia de agroquímicos.

Algumas pesquisas têm demonstrado que certos produtos quando aplicados nas sementes de algumas culturas podem, em determinadas situações, ocasionar redução na germinação e na sobrevivência das plântulas. Por outro lado, alguns inseticidas e fungicidas podem conferir além do efeito protetor, certos tipos de efeitos fisiológicos, auxiliando tanto no crescimento inicial quanto no desenvolvimento das plantas, com possíveis reflexos sobre a produtividade da cultura. Alguns inseticidas podem atuar ativando proteínas transportadoras de membranas celulares, possibilitando o maior transporte iônico, incrementando a nutrição mineral da planta e promovendo respostas positivas no desenvolvimento e na produtividade vegetal (CARVALHO et al., 2011; TAVARES et al. 2013).

O sucesso de uma lavoura é influenciado diretamente pela alta qualidade da semente a ser plantada e contribui significativamente para que níveis de alta produtividade sejam alcançados, enquanto que sementes de baixa qualidade comprometem a obtenção de um estande de plantas adequado, influindo diretamente na produtividade de uma lavoura (KRYZANOWSKI; FRANÇA NETO, 2003).

A qualidade das sementes reflete diretamente no desenvolvimento da cultura, gerando plantas de elevado vigor, uniformidade de população, ausência de doenças transmitidas via semente e maior capacidade competitiva, fatores que podem influenciar diretamente $\mathrm{O}$ rendimento de grãos. Já sementes de baixa qualidade demonstram sintomas típicos de envelhecimento, tais como baixa viabilidade, redução da germinação e taxa de emergência, baixa tolerância a condições sub-ótimas e reduzida taxa de crescimento de plântulas (SILVA; LAZARINI; SÁ, 2010).

Reduções significativas de vigor foram provocadas pelo Carbofuran em sementes de milho, após o tratamento e armazenamento por um período de 30 dias. Sementes de milho 
tratadas com os inseticidas deltametrina $\mathrm{e}$ pirimiphos-methyl, em doses elevadas, conforme Fessel et al. (2003), reduziram a longevidade, o vigor e a velocidade de emergência das plântulas. No tratamento de sementes de milho, reduções no desenvolvimento radicular de plântulas foram provocadas pelo inseticida fipronil (SILVEIRA et al., 2001).

O uso do inseticida Cruiser ${ }^{\circledR}$ formulado à base do ingrediente ativo thiamethoxam, tem seu uso indicado para o tratamento de sementes, como também sua ação comprovada que aponta resultados positivos do mesmo sobre a expressão de características como vigor, germinação, emergência, e aumento de produtividade, em relação às sementes não tratadas (MENTEN, 2005).

O trabalho teve como objetivo avaliar a qualidade fisiológica das sementes de soja (Glycine $\max$ L.) tratadas com os inseticidas Rocks (Bifentrina + Imidacloprido), Cruiser 350 FS (Thiamethoxam), e o fungicida Maxim XL (Metalaxyl-M + Fludioxonil).

\section{MATERIAL E MÉTODOS}

O trabalho foi realizado no laboratório de análise de sementes da Universidade do Estado de Minas Gerais (UEMG). A cultivar utilizada foi a TMG1174RR. Foram avaliados seis tratamentos com quatro repetições de 50 sementes, utilizandose os seguintes produtos: T1- Água Destilada; T2Rocks (Bifentrina + Imidacloprido); T3- Cruiser 350 FS (Tiametoxam); T4- Maxim XL (Metalaxyl$\mathrm{M}+$ Fludioxonil); T5- Rocks + Maxim XL e T6Cruiser ${ }^{\circledR}$ 350+ Maxim XL. As dosagens utilizadas foram determinadas conforme recomendações dos fabricantes e foram às seguintes: Rocks ${ }^{\circledR}$ 0,50 L de produto comercial $100 \mathrm{~kg}-1$ de sementes; Cruiser 350 FS ${ }^{\circledR}$ - 0,25 L de produto comercial 100 kg-1 de sementes e Maxim XL $100 \mathrm{~L}$ de produto comercial $100 \mathrm{~kg}-1$ de sementes. O volume de calda utilizado foi de 300 $\mathrm{mL}$ de calda para $100 \mathrm{~kg}-1$ de sementes para todos os tratamentos realizados.

Para a aplicação dos tratamentos foi preparada uma calda homogênea, a fim de proporcionar o total recobrimento das sementes. A homogeneização da calda com as sementes será realizada em sacos de plástico de $2 \mathrm{~kg}$ de capacidade. O conjunto será agitado por 2 minutos a fim de homogeneizar a cobertura, com posterior secagem a sombra. As sementes foram utilizadas logo após o processo de aplicação dos tratamentos constituídos por inseticidas e/ou fungicida.

As variáveis avaliadas foram: Teste Padrão de Germinação (TPG \%), Índice Velocidade de Emergência (IVE), Condutividade Elétrica da Solução de Embebição (CE) e Comprimento Total de Plântulas (CTP).

\section{Teste Padrão de Germinação (TPG \%)}

Foi realizado em quatro repetições de 50 sementes para cada. As sementes foram colocadas para germinar em substrato de papel de germinação ("germitest"), previamente umedecido em água destilada utilizando-se 2,5 vezes a massa do papel seco, e mantido a temperatura de $25^{\circ} \mathrm{C}$. As avaliações serão efetuadas conforme as Regras para Analise de Sementes (BRASIL, 2009). 


\section{Índice de Velocidade de Emergência (IVE)}

O teste foi realizado a partir da semeadura em vasilhames contendo areia como substrato em quatro repetições de 50 sementes para cada amostra. As plântulas emergidas foram contadas diariamente entre o da emergência e momento de estabilização numérica das contagens. Os resultados foram expressos conforme Maguire (1962).

\section{Condutividade Elétrica da Solução de} Embebição (CE)

Foram utilizadas quatro repetições de 50 sementes de soja, previamente pesadas (0,001 g), colocadas para embeber em copos plásticos (200 $\mathrm{mL}$ ) contendo $75 \mathrm{~mL}$ de água deionizada e mantidas a $25 \quad{ }^{\circ} \mathrm{C} \quad 24 \mathrm{~h}-1$ (HAMPTON; TEKRONY, $\quad 1995 ; \quad$ VIEIRA; KRZYZANOWSKI, 1999). Decorrido o período de embebição, foi relizada a leitura da condutividade elétrica, utilizando-se um condutivímetro DIGIMED, modelo CD 21, com eletrodo de constante 1,0 . Os resultados finais foram expressos em "micro Siemens por centímetro por grama" ( $\mu \mathrm{S} \mathrm{cm}-1 \mathrm{~g}-1)$.

\section{Comprimento Total de Plântulas}

Foram utilizadas quatro subamostras de 15 plântulas para cada tratamento. As sementes foram semeadas em rolos de papel "germitest", umedecido com água destilada, na proporção de 2,5 vezes em relação ao peso seco do papel, e mantidas em germinador regulado a $25{ }^{\circ} \mathrm{C}$ durante sete dias. O comprimento total da plântula (ponta da raiz principal à extremidade da folha mais nova) foi avaliado aos sete dias após a semeadura e os resultados expressos $\mathrm{em} \mathrm{cm}$ por plântula.

Os dados obtidos foram submetidos à Análise de Variância e as médias foram comparadas pelo Teste de Tukey ao nível de 5\% com auxílio do software estatístico SISVAR (FERREIRA, D. F., 2014).

\section{RESULTADOS E DISCUSSÕES}

Os resultados da análise de variância $(0,05)$ e do teste de Tukey $(0,05)$ correspondentes à germinação das sementes, ao índice de velocidade de emergência, à condutividade elétrica e ao comprimento de plântulas estão contidos na tabela 1, onde se verifica diferença estatisticamente significativa apenas para variável comprimento total de plântulas.

Observa-se que as variáveis germinação, índice de velocidade de emergência e condutividade elétrica não apresentaram resultados estatisticamente significativos, ou seja, todos os valores são iguais estatisticamente quando aplicado o teste de Tukey ao nível de probabilidade de $5 \%$. 
Tabela 1. Resultados da análise de variância ao nível de 5\% de probabilidade e do Teste Tukey (5\%)

\begin{tabular}{|c|c|c|c|c|}
\hline \multirow{2}{*}{ Tratamentos } & \multicolumn{4}{|c|}{ Variáveis Avaliadas } \\
\hline & $G(\%)$ & IVE & $\mathrm{CE}$ & CTP \\
\hline 1 Água destilada & 92,00 & 10,51 & 64,58 & $15,98 \mathrm{~b}$ \\
\hline 2 Bifentrina + Imidacloprido & 84,00 & 10,13 & 64,85 & $15,17 \mathrm{~b}$ \\
\hline 3 Thiamethoxam & 90,00 & 10,84 & 57,42 & $22,37 \mathrm{a}$ \\
\hline 4 Fludioxonil + Metalaxyl-M & 91,50 & 11,42 & 56,93 & $21,59 \mathrm{a}$ \\
\hline $5 \begin{array}{l}\text { Bifentrina + Imidacloprido e } \\
\text { Fludioxonil + Metalaxyl-M }\end{array}$ & 88,00 & 11,18 & 66,21 & $20,08 \mathrm{a}$ \\
\hline $6 \begin{array}{l}\text { Thiamethoxam e } \\
\text { Fludioxonil + Metalaxyl-M }\end{array}$ & 89,00 & 10,34 & 70,45 & $21,65 \mathrm{a}$ \\
\hline $\mathrm{F}$ & 2,82 n.s. & 3,09 n.s. & 2,52 n.s. & $38,94^{*}$ \\
\hline $\mathrm{CV}(\%)$ & 3,93 & 5,31 & 11,06 & 5,14 \\
\hline DMS & - & - & - & 2,30 \\
\hline
\end{tabular}

G (\%) - Germinação; IVE - índice de Velocidade de Emergência; CE - Condutividade Elétrica da Solução de Embebição; CTP - Comprimento total de Plântulas.

n.s. - não significativo ao nível de $5 \%$ de probabilidade pelo teste $\mathrm{F}$; * significativo ao nível de $5 \%$ pelo teste $\mathrm{F}$.

Médias seguidas pela mesma letra não diferem entre si pelo teste de Tukey ao nível de $5 \%$ de probabilidade.

A germinação é um fator de grande importância, uma vez que, quando as sementes apresentam uma baixa porcentagem de germinação isso ocasiona em falhas no estande de plantas e um provável prejuízo aos produtores. Segundo Brasil (2005), em que é definido que a porcentagem mínima de germinação deve se de 80\%. Para todos os tratamentos avaliados a porcentagem de germinação foi satisfatória do ponto de vista legislativo, pois em todos os tratamentos a germinação foi superior a $80 \%$.

O índice de velocidade de emergência foi outra variável em que se notou uma diferença estatisticamente significativa em função dos tratamentos utilizados. Grisi et al. (2009), estudando o efeito de inseticidas no tratamento de sementes de girassol, não constataram alteração no vigor e na emergência das sementes tratadas com thiamethoxam e fipronil.

No tratamento de sementes de feijão com os inseticidas imidacloprido e thiamethoxam, os ingredientes ativos proporcionaram melhoria nas características agronômicas da cultura, resultando em aumento de produtividade (DAN, L. G. M, et al, 2010; BARBOSA et al., 2012). Barros et al. (2005), citado por Dan et al. (2010), verificaram maior porcentagem de germinação das sementes de feijão nos tratamentos com o inseticida fipronil (DAN, L. G. M, et al, 2010; BARBOSA et al., 2012). De acordo com Melo et al. (2010), em relação a germinação na cultura do milho, os tratamentosFipronil e Thiamethoxam não diferiram estatisticamente, sendo que a testemunha foi estatisticamente superior a estes tratamentos.

Em um estudo realizado por Dan et al. (2010) constatou-se que ao final de um período de armazenamento de 45 dias os tratamentos com os inseticidas fipronil, thiamethoxam, imidacloprido, e imidacloprido + thiodicarb apresentaram ainda percentuais de germinação acima de 80\% evidenciando que os tratamentos não influenciaram na germinação mesmo após decorrido um período de armazenamento. Neste 
mesmo estudo pode-se observar que os tratamentos utilizados também não influenciaram no vigor das sementes que foi determinado através da velocidade de emergência.

Segundo Vieira et al. (2002), a condutividade elétrica é um parâmetro que visa avaliar a integridade das membranas celulares através da determinação da quantidade de solutos lixiviados para a solução de embebição. Com isso, pode-se observar que sementes com maior vigor tentem a possuir valores menores de condutividade elétrica devido ao fato de estas possuírem maior integridade das membranas celulares. O fato de os valores de condutividade elétrica não terem diferenciado entre si estatisticamente é um indicativo de que os tratamentos aplicados não influenciaram no processo de degradação celular que tem como um de seus primeiros processos observados a desorganização do sistema de membranas celulares.
No trabalho de Melo et al. (2010), os valores de condutividade elétrica na cultura do milho diferiram estatisticamente, sendo que a menor lixiviação foi no tratamento testemunha, e os demais tratamentos: Thiamethoxam e Fipronil não apresentaram diferenças estatísticas entre si, entretanto, não levaram a redução do vigor das sementes permanecendo com alto vigor.

Quando analisada a variável comprimento de plântulas demonstrou que houve diferenças estatisticamente significativas, sendo que os tratamentos 3 (Cruiser 350 FS®), 4 (Maxim XL®), 5 (Rocks ${ }^{\circledR}+$ Maxim XL $\left.®\right)$, e 6 (Cruiser 350 FS ${ }^{\circledR}+$ Maxim XL $\left.{ }^{\circledR}\right)$ demonstraram propiciar um maior desenvolvimento inicial das plântulas quando comparado com os tratamentos que receberam apenas a aplicação de água destilada e o inseticida Rocks ${ }^{\circledR}$. Estes resultados podem ser observados na figura 1.

\section{Comprimento Total de Plantulas}

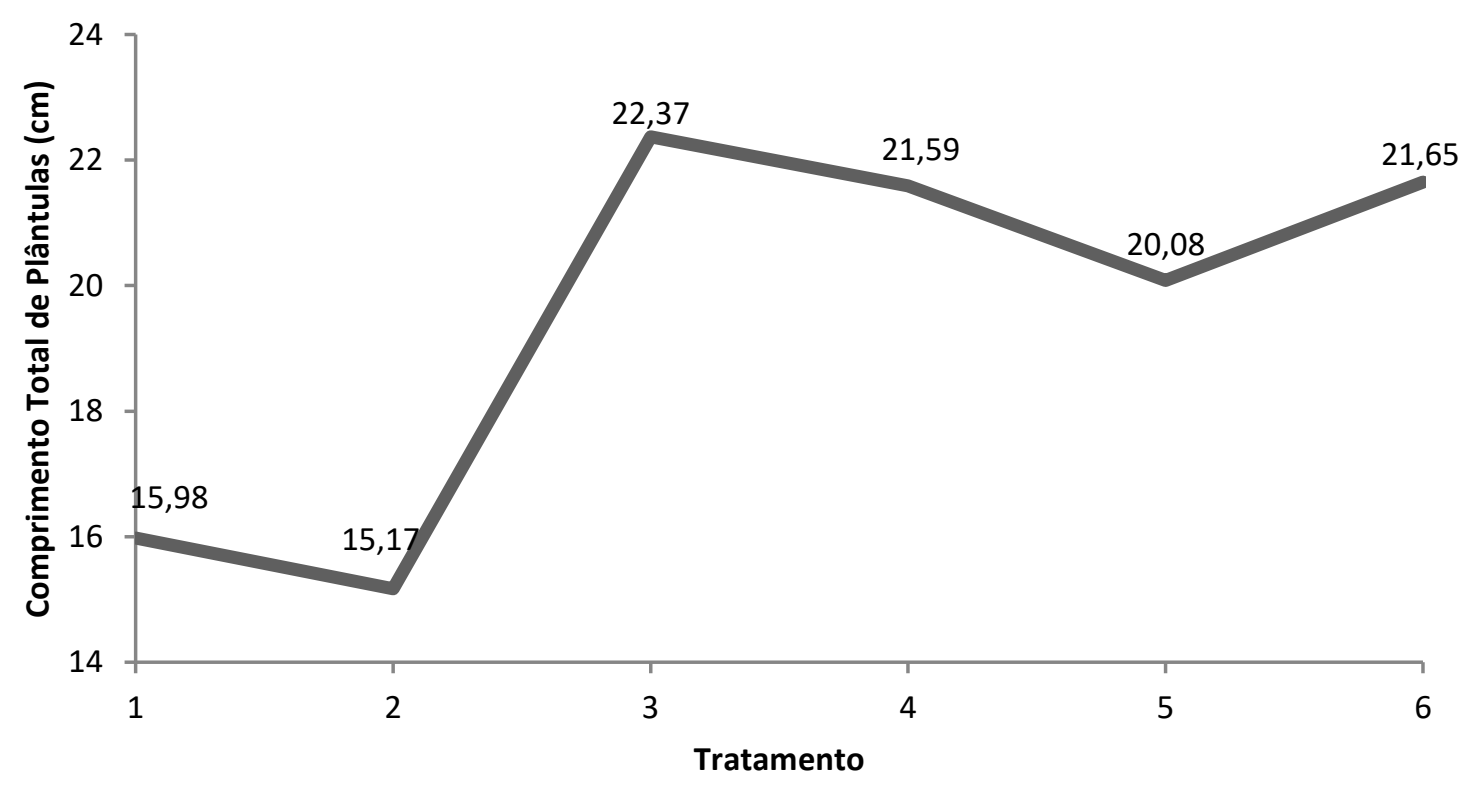

Figura 1. Comprimento de plântulas em função dos tratamentos aplicados. 
Segundo Carvalho et al. (2011) citado por Tavares et al. (2013), Alguns inseticidas podem atuar ativando proteínas transportadoras possibilitando o maior transporte iônico, promovendo respostas positivas no desenvolvimento e na produtividade vegetal. Este fator pode explicar o maior crescimento das plântulas que receberam o tratamento com inseticidas e fungicidas em comparação com o tratamento que recebeu apenas água destilada.

Dan et al. (2010), avaliando o efeito de inseticidas sobre o desenvolvimento de plântulas de soja observou uma maior redução do comprimento de plântulas de soja submetidas ao tratamento de semente com o inseticida thiamethoxam.

Guimarães et al. (2005) observaram que o inseticida thiamethoxam afetou negativamente a altura das plântulas na cultura de feijão preto. Sendo que, Tavares et al. (2007) não encontraram diferença no desenvolvimento de plântulas de soja, no tratamento de sementes com cinco doses de thiamethoxam.

De acordo com Melo et al. (2010), os resultados de índice de velocidade de germinação diferiram estatisticamente na cultura do milho, o Thiamethoxam apresentou valores superiores em relação à Fipronil e testemunha. No trabalho de Dan et al. (2010), para a característica de velocidade de emergência não foi observado diferença significativa entre a testemunha e os tratamentos tratados com Thiamethoxam e Fipronil, onde que indica que os inseticidas não afetaram nas sementes enquanto que em um trabalho realizado por Grisi et al. (2009), os tratamentos thiamethoxam e fipronil não observaram diferenças estatísticas na emergência das sementes de girassol.

\section{CONCLUSÕES}

A aplicação dos inseticidas e do fungicida não influenciou na germinação das sementes de soja. Com relação à qualidade fisiológica das sementes entre todas as variáveis avaliadas apenas o comprimento total de plântulas foi influenciado pela aplicação dos tratamentos compostos por dois inseticidas e um fungicida.

\section{REFERÊNCIAS BIBLIOGRÁFICAS}

BRASIL. Ministério da Agricultura, Pecuária e Abastecimento. Regras para análise de sementes. Ministério da Agricultura, Pecuária e Abastecimento. Secretaria de Defesa Agropecuária. Brasília, DF: Mapa/ACS, 2009. $398 \mathrm{p}$.

BULEGON, L. G.; CASTAGNARA D. D.; TSUTSUMI, C. Y.; ERIG, M.C.; ZOZ, T. Germinação e emergência de sementes de diferentes tamanhos submetidas à tratamentos químicos. Revista de Agricultura Neotropical, Cassilândia-MS, v. 2, n. 2, p. 86-94, abr./jun. 2015.

DAN, Lilian Gomes de Moraes et al. QUALIDADE FISIOLÓGICA DE SEMENTES DE SOJA TRATADAS COM INSETICIDAS SOB EFEITO DO ARMAZENAMENTO. Revista Brasileira de Sementes, Brasil, v. 32, n. 2, p.131-139, 2010.

DARÉ, L., CATANEO, A. C. Melhoria da germinação de sementes de soja sob estresse salino através do aumento da atividade da enzima peroxidase. Universidade Estadual Paulista Júlio de Mesquita Filho: $16^{\circ}$ Congresso de Iniciação Cientifica. 9 p, 2016.

FERREIRA, D. F. SISVAR: a Guide for its Bootstrap procedures i multiple comparisons. Ciênc. Agrotec, vol. 38, n.2, p.109-112, 2014. Disponível em: htpp://dx.doi.org/10.1590/S141370542014000200001 . 
GUIMARÃES, R.N.; PORTO, T.B.; PEREIRA, J.M.; BARBOSA, L.A.; FERNANDES, P.M.; COSTA, R.B.; BARROS, R.G. Efeito do Tratamento de Sementes com Inseticidas na Emergência e Altura de Plântulas de Feijão. In: CONGRESSO NACIONAL DE PESQUISA DE FEIJÃO, 8, 2005, Goiânia. Anais... Goiânia: Embrapa Arroz e Feijão, 2005. p.9499. (Embrapa Arroz e Feijão. Documentos, 182).

GRISI, P.U.; SANTOS, C.M.; FERNANDES, J.J.; SÁ JÚNIOR, A. Qualidade das sementes de girassol tratadas com inseticidas e fungicidas. Bioscience Journal, v.25, n.4, p.2836, 2009.

HENNING, A. A.; KRZYZANOWSKI, F. C.; FRANÇANETO, J. B.; YORINORI, J.T. Tratamento de sementes de soja com fungicida. Londrina: EMBRAPA-CNPSo, 1991. 4 p. (EMBRAPA-CNPSo. Comunicado Técnico,49).

MAGUIRE, J. D. Speed of germination-aid selection and evaluation for seedling emergence and vigor. Crop Science, v. 2, n. 2, p. 176-177, 1962.

MELO, L. F.; FAGIOLI, M.; SUSSTRUNK, T. F. Tratamento de sementes de milho com fipronil e thiamethoxam e sua influência fisiológica nas sementes. Agropecuária Técnica, v. 31, n. 2, p. 49-56, 2010.

MENTEN, O.J. Tratamento de sementes no Brasil. SEEDNews.Pelotas/ RS, n.5, p.30-3132, (setembro/outubro), 2005.

SILVA, J. B.; LAZARINI, E.; SÁ, M. E. Comportamento de sementes de cultivares de soja, submetidos a diferentes períodos de envelhecimento acelerado. Bioscience Journal, Uberlândia, v. 26, n. 5, p. 755-762, 2010.

SILVEIRA, R.E.; MACCARI, M.; MARQUEZI, C.F. Avaliação do efeito de inseticidas aplicados via tratamento de sementes sobre o desenvolvimento de raízes de milho, na proteção de pragas do solo. In: REUNIÃO SUL-BRASILEIRA SOBRE PRAGAS DE SOLO, 8., 2001, Londrina. Anais... Londrina: Embrapa Soja, 2001. p. 246-249.

TAVARES, L. C., MENDONÇA, A. D., ZANATTA, Z. C. N., BRUNES, A. P., \& VILLELA, F. A. Efeito de fungicidas e inseticidas via tratamento de sementes sobre o desenvolvimento inicial da soja. Enciclopédia biosfera, Centro Científico Conhecer, v. 10, n. 18, p. 1400-1409, 2014.

TAVARES, S.; CASTRO, P.R.C.; RIBEIRO, R.V.; ARAMAKI, P.H. Avaliação dos efeitos fisiológicos de thiametoxan no tratamento de sementes de soja. Revista de Agricultura, v.82, n.1, p.47-54, 2007.

VIEIRA, Roberval Daiton et al. Condutividade elétrica e teor de água inicial das sementes de soja. Pesq. Agropec. Bras., Brasília, v. 37, n. 9, p.1333-1338, set. 2002.

VIEIRA,R. D., Penariol, A. L., Perecin, D., \& Panobianco, M. Electrical conductivity and initial water content of soybean seeds. Pesquisa Agropecuária Brasileira, v. 37, n. 9, p. 13331338, 2002.

VIEIRA, R.D.; KRZYZANOWSKI, F.C. Teste de condutividade elétrica. In: KRZYZANOWSKI, F.C.; VIEIRA, R.D.; FRANÇA NETO, J.B. (Ed.). Vigor de sementes: conceitos e testes. Londrina: ABRATES, 1999. p.4-1 a 4-26. 